\title{
La cultura como estrategia de transformación y promoción urbana en Bogotá y Medellín ${ }^{1}$
}

\author{
Isabel Duque Franco ${ }^{2}$
}

\begin{abstract}
RESUMEN
En décadas recientes, diferentes ciudades en el mundo han emprendido procesos de planeamiento urbano utilizando la cultura como principal estrategia. Este artículo, a partir del concepto de planeamiento urbano cultural, explora el papel que ha desempeñado la cultura en la transformación urbana de las ciudades de Bogotá y Medellín durante las últimas dos décadas y que les ha permitido alcanzar un cierto reconocimiento internacional. Con base en el estudio de diferentes políticas, planes y proyectos, el artículo muestra cómo ha sido abordada la cultura en cada caso, los repertorios de intervención urbanística asociados a la cultura y las estrategias de promoción internacional de ambas ciudades como centros culturales.
\end{abstract}

Palabras clave: Cultura, planeamiento urbano, gobernanza urbana, Bogotá, Medellín

\begin{abstract}
In recent decades, different cities around the world have undertaken urban planning processes that focus on culture as their main strategy. This article, based on the concept of cultural urban planning, explores the role that culture has played in the urban transformation of the cities of Bogota and Medellin over the last two decades, which has brought them international recognition. By studying various policies, plans and projects, the paper analyzes how culture has been addressed in each case, the repertories of urban intervention associated with culture and the strategies used by both cites for their international promotion as cultural centers.
\end{abstract}

Key words: Culture, urban planning, urban governance, Bogota, Medellin.

\footnotetext{
Este trabajo hace parte de la investigación "Cultura y ocio en el planeamiento urbano de Bogotá y Medellín" financiada por la Facultad de Ciencias Humanas de la Universidad Nacional de Colombia en el marco de la Convocatoria de Investigación Orlando Fals Borda 2011 y contó con el apoyo de
}

Manuel Camilo Velandia. Artículo recibido el 2 de julio de 2014, aceptado el 5 de diciembre de 2014 y corregido el 4 de marzo de 2015.

2 Universidad Nacional de Colombia (Colombia). Email: miduquef@unal.edu.co 
Durante las últimas dos décadas, en la mayoría de ciudades del mundo, se han venido desarrollando diversos planes, proyectos y políticas urbanas que utilizan la cultura como principal estrategia (UN-Habitat, 2004). Ya sea mediante la preservación de centros históricos, la reconversión de infraestructura obsoleta en equipamientos culturales, el cuidadoso diseño de espacios públicos o la promoción de industrias culturales; cada vez se hace más evidente el esfuerzo de los gobiernos urbanos por apelar a la cultura como recurso a la hora de identificar los aspectos diferenciales de las ciudades, sus ventajas comparativas y su singularidad, lo que las ha llevado a rescatar lugares simbólicos y representativos o simplemente a inventarlos. En definitiva, la cultura se ha instalado en la agenda urbana y cada vez son más las políticas orientadas hacia la construcción de una imagen de ciudad basada en la cultura, el ocio y el turismo como claves de diferenciación y competitividad (Evans, 2003; Bayliss, 2004; 2007; Manito, 2006; 2011).

Estas tendencias globales en el planeamiento urbano, han migrado hacia algunas ciudades colombianas, que las han ido adaptando a las especificidades locales. Tal es el caso de Bogotá y Medellín que, durante los últimos años, han sido reconocidas a nivel internacional por haber emprendido ambiciosos procesos de reestructuración y planificación que incluyen ingeniosas alternativas en movilidad, políticas para la reducción de la pobreza, diseño de espacios públicos como parques y plazas y construcción de equipamientos culturales como museos y bibliotecas. En este sentido, este artículo tiene como objetivo examinar cómo ha sido la instrumentalización de la cultura en los procesos de transformación y promoción urbana en Bogotá y Medellín durante las dos últimas décadas. Para ello, luego de la contextualización acerca de la relación entre cultura, creatividad y planeamiento, se aborda la experiencia de las dos ciudades desde tres categorías de análisis: los enfoques y perspectivas desde las cuales se aborda la cultura; los repertorios del planeamiento cultural urbano, es decir, el tipo de intervenciones urbanísticas a través de los cuales se materializan los enfoques (Manito, 2006); y por último, las estrategias de promoción internacional con las cuales se pretende posicionar a estas ciudades como centros culturales, que suelen ser campañas de marketing urbano, participación en redes de ciudades asociadas a la cultura o la organización de eventos de amplio reconocimiento (Gold \& Ward, 1994; Benko, 2000).

\section{Cultura, creatividad y planeamiento urbano}

El posicionamiento de la cultura como estrategia de planeamiento urbano está asociado al paso de la ciudad máquina a la ciudad objeto de consumo (Arantes, 2000). Los procesos de desindustrialización y de reestructuración, sumados a los cambios en la composición tecnológica de la producción, al aumento de la movilidad del capital y a los nuevos productos y mercados, provocaron la pérdida de las ventajas competitivas de los otrora centros industriales, dando lugar a una nueva relación entre las ciudades, determinada básicamente por la necesidad de competir entre sí como centros financieros, de consumo y de entretenimiento para atraer y fijar un capital que es cada vez más volátil (Harvey, 1989).

En este marco de competitividad y tercerización de la economía, las ciudades han encontrado en la cultura un campo de infinitas posibilidades. "La cultura es cada vez más el negocio de las ciudades, la base de sus atracciones turísticas y su única ventaja competitiva" (Zukin, 1995: 2), en tanto puede contribuir a la reactivación de la economía mediante el desarrollo de las industrias culturales y al mismo tiempo servir para proyectar una nueva imagen de ciudad basada en la cultura y la creatividad, atractiva para turistas e inversores.

Desde el ámbito del planeamiento y la gobernanza urbana, aquellas ciudades capaces de identificar la cultura como fuente de diferenciación e innovación, de creación de valor social y económico, han sido denominadas "ciudades creativas" (Manito, 2011). Se trata de ciudades que articulan el patrimonio, los productos y servicios culturales tradicionales con las industrias creativas y que proyectan un estilo de vida dinámico, atractivo y diverso. Este concepto de la ciudad creativa fue introducido por Charles Landry y Franco Bianchini (1995) y luego popularizado por 
Richard Florida y su teoría de la clase creativa (2005). En principio, la ciudad creativa era una aspiración, un llamado al uso de la imaginación y la innovación en la solución de los problemas urbanos derivados del paso de una economía basada en las manufacturas a una economía centrada en el conocimiento, sin embargo, la creatividad aplicada a la planificación de las ciudades involucra un potencial mucho mayor, asociado a intervenciones urbanísticas, modelos de gobernanza y de toma de decisiones (Landry, 2000).

La articulación entre planeamiento urbano, cultura y creatividad se ha traducido en un repertorio de proyectos, que responden a la lógica de los flujos y la movilidad de políticas urbanas, es decir que migran de un lugar a otro y en el proceso se transforman, mutan y se adaptan a las particularidades de cada ciudad (Peck \& Theodore, 2010; Peck, 2011). Según los planteamientos de Landry (2000), UN-Habitat (2004) y Manito (2006) estos proyectos urbanos se pueden clasificar en cuatro tendencias complementarias. En primer lugar, están las "iniciativas de renovación y revitalización de espacios urbanos", con ejemplos emblemáticos como Glasgow con su paso de ciudad industrial en decadencia a capital europea de la cultura (Gómez, 1998; Evans \& Shaw, 2004); Bilbao con el polémico "efecto Museo Guggenheim" (Arantes, 2000; González, 2004; Plaza, 2006); Ciutat Vella de Barcelona, en donde convergen todas las intervenciones posibles en términos de renovación urbana (Capel, 2005); la rehabilitación de Puerto Madero en Buenos Aires (Cuenya y Corral, 2011) o más recientemente el polémico megaproyecto Puerto Maravilla en Río de Janeiro (Lima, 2010; Castro Coma, 2011). En general, se trata de proyectos de reconversión funcional y desarrollo de nuevos usos urbanos, gestionados por asociaciones públicoprivadas, que involucran la subvención de iniciativas privadas con recursos públicos, lógicas de especulación inmobiliaria, generación de beneficios económicos y pérdida de control por parte de los actores estatales.

En segundo lugar, se encuentran los proyectos relacionados con las "industrias culturales o creativas". A pesar de la popularidad y el interés despertado por el concepto, diferentes autores coinciden en señalar su indefinición y la confusión conceptual que existe sobre el tema, lo que dificulta establecer qué actividades pueden ser consideradas como industrias creativas o culturales (Bayliss, 2007; Méndez et al., 2012; Scott, 2010). En cualquier caso, diferentes trabajos muestran cómo la concentración de estas actividades ha llevado a la configuración de distritos culturales, bien sea de manera espontánea o dirigida a través de políticas públicas. Algunos ejemplos son el Temple Bar en Dublín (Bayliss, 2004); el complejo cinematográfico de Los Ángeles, el distrito cerámico de Caltagirone en Sicilia (Santagata, 2002); San Telmo en Buenos Aires (Ramón, 2011); el Raval de Barcelona y el Mile End de Montreal (Battaglia \& Tremblay, 2012).

En tercer lugar, está la conformación de "ejes o corredores culturales", espacios públicos abiertos, con una oferta material y simbólica que incluye lugares de ocio y equipamientos culturales, en algunos casos con cierto grado de especialización y con una oferta comercial combinada de vivienda y servicios (Manito, 2006). Algunos de estos corredores se han consolidado históricamente como la avenida Corrientes en Buenos Aires; otros son el resultado de intervenciones de renovación urbana en zonas portuarias y waterfronts como en Liverpool, Londres y Buenos Aires (Evans \& Shaw, 2004; Muñoz, 2008). En otros casos, estos corredores surgen como conectores de infraestructura cultural o por la creación de nuevas centralidades con el diseño de espacios públicos en torno a grandes equipamientos (Manito, 2006).

Finalmente, está la formulación de "políticas culturales" que, como complemento a una cierta infraestructura física, incluyen una agenda cultural permanente y diversa con exposiciones, conciertos, ferias y festivales; aunque también algunos de estos eventos tienen como finalidad dinamizar procesos de regeneración urbana y construir nuevos equipamientos (García, 2004; Benneworth \& Dauncey, 2010; Devesa et al., 2012). Como estos eventos son ofertas puntuales, que resultan atractivas solamente durante su realización, crece el afán de las ciudades por crear nuevas ferias y festivales que resulten atractivas para turistas y visitantes. Sin embargo, según Landry (2000), las políticas culturales también pueden estar orientadas al fortalecimiento de la cohesión social, la creatividad 
y la capacidad de las personas para actuar como ciudadanos democráticos.

Aunque el planeamiento cultural ha sido acogido con entusiasmo por diferentes gobiernos urbanos, se trata de una tendencia que suscita importantes conflictos y dilemas. De un lado, las estrategias de revitalización urbana excluyen los grupos de bajos ingresos y generan procesos de gentrificación. Este fenómeno ha sido ampliamente estudiado en ciudades como Nueva York (Zukin, 1995) o Londres (Muñoz, 2008). Recientemente Janoschka y Sequera (2014) lo han abordado también en México, Buenos Aires y Río de Janeiro, en donde los proyectos de recuperación del centro histórico, la creación de "polos de desarrollo cultural" o la adecuación urbana para los megaeventos, han promovido desarrollos inmobiliarios que fomentan la progresiva expulsión de sectores populares del centro de estas ciudades.

De otro lado, la competitividad urbana exige a las ciudades la capacidad de ofrecer lugares con cualidades únicas y especiales, lo suficientemente atractivos como para diferenciarse de las demás ciudades con las que compiten. Sin embargo, como se ha visto en el caso del planeamiento cultural, los repertorios de actuación están prácticamente establecidos, de manera que alcanzar la singularidad no solo resulta cada vez más difícil, sino que además, las mismas estrategias circulan de una ciudad a otra, produciendo versiones de lo mismo o en el peor de los casos, imágenes repetidas y estandarizadas (Harvey, 1989; Muñoz, 2008).

Esta breve revisión sobre las sinergias entre cultura, creatividad y planeamiento urbano, permite ver que la cultura es entendida como un sector económico relacionado con las industrias creativas y culturales, y al mismo tiempo como un recurso para la reconstrucción de la imagen de las ciudades con el fin de atraer empresas, inversiones y turistas (Bayless, 2007). Este trabajo se centra fundamentalmente en esta segunda perspectiva. De otra parte, la revisión también permite evidenciar que el planeamiento cultural no es un fenómeno exclusivo de las ciudades del capitalismo industrial y que tampoco resulta ajeno al contexto latinoamericano. En este sentido, los casos de Bogotá y Medellín no son expe- riencias aisladas, ni simples imitaciones de las políticas urbanas desplegadas en otros lugares, sino un ejercicio de recontextualización de esta forma de planeamiento. De ahí el interés en este trabajo por evidenciar los rasgos del proceso de inclusión de la cultura en el planeamiento urbano de estas dos ciudades.

\section{Bogotá: de la cultura ciudadana a la cultura mercancía}

Al comenzar la década de los noventa, Bogotá arrastraba serios problemas derivados del continuo crecimiento físico y demográfico, la ausencia de un liderazgo fuerte y los profundos problemas de corrupción. El deterioro que presentaba la ciudad se expresaba en: elevados niveles de pobreza $(42,4 \%)$, un sistema de transporte caótico, los pocos espacios públicos existentes habían sido privatizados o carecían de mantenimiento y el centro histórico estaba abandonado y degradado. A todo esto había que sumar la inseguridad que vivía la ciudad, reflejada en tasas de homicidio que en 1993 habían alcanzado los 80 por cada cien mil habitantes (Martín y Ceballos, 2004; PNUD, 2008).

Desde entonces, aunque no todas estas problemáticas han podido resolverse completamente, Bogotá no solo ha cambiado físicamente, sino que ha experimentado transformaciones en las formas de vivir, percibir, planear y gobernar la ciudad. Las diferentes administraciones que ha tenido Bogotá durante este tiempo, han configurado un modelo de ciudad que se caracteriza por la continuidad como opción de gobierno, la variedad de enfoques orientadores de la gestión y la intervención sobre temas fundamentales como el espacio público o la movilidad (Duque Franco, 2008).

\section{Enfoques y perspectivas en torno a la cultura}

A partir del análisis de los diferentes instrumentos de planeación formulados e implementados en Bogotá entre 1995 y 2011, se identifica que la relación entre cultura y planeamiento urbano ha sido abordada desde cuatro perspectivas: la cultura asociada al comportamiento, es decir, la cultura ciuda- 
dana; la cultura como una dimensión de la ciudad a escala humana que se traduce en equipamientos y espacio público; la cultura desde la perspectiva de los derechos civiles, económicos, sociales y culturales (DESC) y finalmente, la cultura como estrategia de competitividad urbana.

A mediados de la década de 1990, surgía la visión de la ciudad como problemática cultural en tanto dimensión de la existencia individual y colectiva. La apuesta por la cultura ciudadana estaba sustentada en la hipótesis, formulada por el propio alcalde, según la cual gran parte de los problemas que tenía la ciudad estaban motivados por los comportamientos violentos y que estos a su vez, se explicaban por el divorcio existente entre los tres sistemas reguladores del comportamiento humano: ley, moral y cultura (Mockus, 2001:17-35). Existe divorcio cuando hay aprobación cultural (colectiva) y/o moral (individual) de las acciones ilegales y cuando no hay aprobación moral o cultural de las obligaciones legales.

Siguiendo esta premisa, se consideró que a través de la comunicación y de la interacción intensificada en el espacio público, era posible reducir este divorcio. Así, se promovió un conjunto de acciones con el propósito de, primero, construir colectivamente una imagen de ciudad actual y futura; segundo, buscar que la comprensión y el respeto de las reglas generara identidad ciudadana y sentido de pertenencia; tercero, impulsar la cultura popular y las manifestaciones artísticas y por último, modificar los comportamientos individuales y colectivos que reñían fuertemente con la vida social de la ciudad, a través de la autorregulación ciudadana y del rediseño y construcción de algunos espacios urbanos en los cuales interactuaban los ciudadanos.

Esta relación entre cultura ciudadana y espacio urbano, empezó a cobrar mayor sentido a finales de la década de los noventa con la fuerte campaña de recuperación, mejoramiento y ampliación del espacio público: alamedas, plazoletas, ejes urbanísticos, parques y ciclo rutas e hitos urbanos derivados de procesos de renovación urbana y de construcción de equipamientos como la Red Capital de Bibliotecas Públicas. Estas intervenciones urbanísticas estaban inspiradas en la necesi- dad de construir una ciudad a escala humana, pensada y centrada en las personas, en donde el espacio público se convirtiera en escenario para el espectáculo, el arte, la cultura y las diferentes manifestaciones creativas de los ciudadanos (Concejo de Bogotá, 1998).

Posteriormente, desde mediados de la década del 2000, el enfoque integral de derechos humanos se convirtió en el principio orientador de la acción de la administración bogotana y el acceso a la cultura, en sus diferentes expresiones, fue considerado como un derecho. Desde esta perspectiva, se formularon una serie de políticas públicas como el Plan Maestro de Equipamientos Culturales (PlaMEC), las Políticas Culturales Distritales 2004-2016 y el Plan Decenal de Cultura 2012-2021, que le concedieron especial importancia a la descentralización y democratización de la oferta de bienes y servicios culturales, a la creación del Sistema Distrital de Cultura $^{3}$ y al estímulo a las iniciativas de formación, creación y circulación artística, así como a las diferentes expresiones culturales, "porque el ejercicio de los derechos culturales no se limita tan solo a reclamar el acceso a la cultura 'de otros', sino que se extiende al derecho de desplegar la de cada cual en igualdad de condiciones" (Alcaldía Mayor de Bogotá, 2005:11).

Aunque tanto el PlaMEC como el Plan Decenal de Cultura se inspiraban en el enfoque de derechos, también insistían en el potencial competitivo y económico de la cultura y en la necesidad de posicionar a la ciudad en la escena iberoamericana y mundial como epicentro cultural. Algunos lineamientos del Plan como la identificación y diversificación de las industrias culturales, el impulso al desarrollo de nuevas tecnologías de la información y comunicación dentro del sector y el fortalecimiento de las cadenas de valor en las prácticas culturales, las artes y el patrimonio cultural, ponen de manifiesto la asimilación de la cultura como generadora de riqueza y también la intención de avanzar en la idea de

\footnotetext{
3 Compuesto por un conjunto de espacios, instancias, procesos y dimensiones que buscan interpretar e intervenir la cultura como un campo donde se articulan de manera variada actividades, profesiones, instituciones y agentes culturales.
} 
Bogotá como ciudad creativa (Alcaldía Mayor de Bogotá, 2011).

En términos generales, las diferentes perspectivas con las que se ha abordado la cultura en Bogotá no han sido ajenas al problema de la competitividad, y en ese sentido, han estado acompañadas, en mayor o menor medida, por la idea de la cultura como activo y como estrategia para hacer la ciudad más atractiva para inversores, visitantes y turistas, crear una imagen de Bogotá como referente cultural y como tal, posicionarla en el contexto global (Alcaldía Mayor de Bogotá D.C., 2011).

\section{Repertorios del planeamiento cultural urbano}

En Bogotá, la articulación entre la cultura y el planeamiento urbano se ha traducido en la revalorización de la infraestructura existente (museos, teatros, galerías) y la construcción de nuevos espacios públicos y equipamientos, destinados a armonizar el mejoramiento de la calidad de vida de los bogotanos con la promoción internacional de la ciudad. En este sentido, durante las últimas dos décadas se han desarrollado dos tipos de intervenciones urbanísticas asociadas a la cultura: la construcción de nuevos equipamientos y la configuración de corredores culturales en el marco de procesos de renovación urbana (Figura $\mathrm{N}^{\circ} 1$ ).

De acuerdo con esto, fueron construidas cuatro megabibliotecas en sitios estratégicos de la ciudad e integradas a otros espacios públicos El Centro Cultural Biblioteca Julio Mario Santodomingo ubicado en el norte de la ciudad. La Biblioteca Virgilio Barco está en

Figura $N^{\circ} 1$

Localización de equipamientos culturales en Bogotá

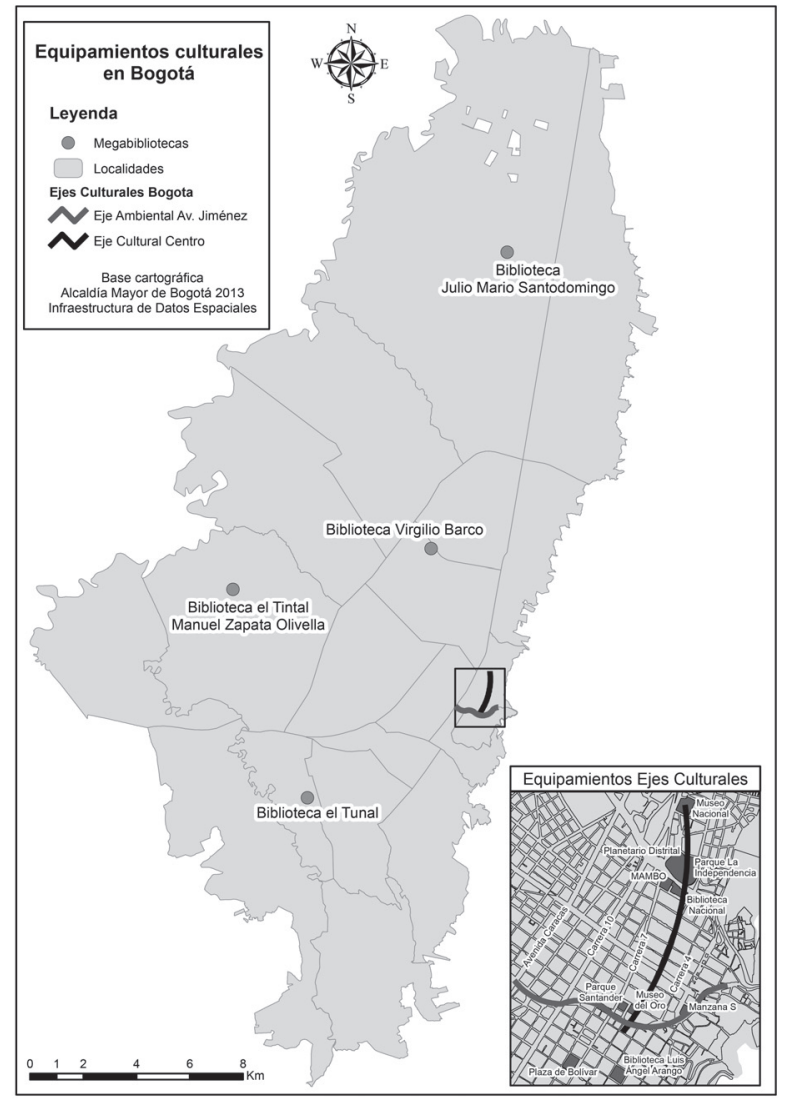

Fuente: Elaborado por Wendy González. 
el costado del Parque Metropolitano Simón Bolívar, es una obra del arquitecto colombiano Rogelio Salmona, que se ha convertido en todo un referente en términos culturales y arquitectónicos (Figura № 2). La Biblioteca El Tintal resultado de la reconversión de una antigua planta recicladora, ha sido una pieza fundamental en la configuración de una centralidad urbana en el suroccidente de la ciudad (Beuf, 2012 ) (Figura N 3). Por último, la Biblioteca El Tunal construida en el parque del mismo nombre al sur de la ciudad. Estas dos últimas se encuentran en zonas donde predominan los sectores de bajos ingresos y medios, en menor proporción. Estos equipamientos son ambientes multifuncionales que han contribuido a enriquecer y diversificar la oferta cultural, educativa y de espacio público en la ciudad.

El PlaMEC planteaba la construcción de equipamientos culturales de alta jerarquía en el centro y en las demás centralidades de Bogotá, con el propósito de conformar una Red de Infraestructura Territorial Cultural para la creación, investigación, formación, circulación y apropiación del arte, la cultura, el patrimonio, la ciencia y la tecnología (Alcaldía Mayor de Bogotá, 2006). Sin embargo, en 2011 se advirtió que la posibilidad de construir nuevos equipamientos culturales, que sirvieran de elementos articuladores del modelo territorial, estaba seriamente condicionada por la disponibilidad de suelo no solo en el centro sino en el resto de la

Figura $\mathrm{N}^{\circ} 2$

Biblioteca Virgilio Barco Vargas

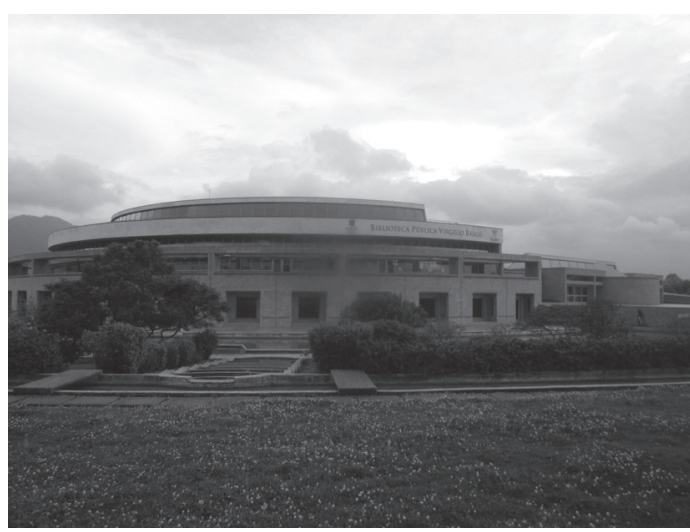

Fuente: Manuel Velandia, 2012. ciudad. Atendiendo a esta dificultad, la administración de la ciudad a través del Instituto Distrital de Cultura, diseñó tres estrategias: extender las actividades culturales a los equipamientos educativos y recreativos; hacer un uso intensivo de los equipamientos culturales existentes y establecer alianzas públicoprivadas para el uso de la infraestructura cultural privada (Alcaldía Mayor de Bogotá D.C., 2011).

Por otra parte, dentro del repertorio del planeamiento cultural en la ciudad, está también la consolidación de rutas o corredores culturales, como parte de los procesos de renovación urbana ligados al Plan Zonal del Centro. En este sentido destacan la reutilización de edificaciones para fines turísticos y culturales, así como la construcción de espacios nuevos con inversión privada, como el centro cultural mexicano Gabriel García Márquez del Fondo de Cultura Económica de México en La Candelaria (Figura $N^{\circ} 4$ ), que complementa y diversifica la oferta cultural del sector y al mismo tiempo constituye un nuevo hito arquitectónico y paisajístico, que en un solo eje articula la Plaza de Bolívar, la Catedral, la Casa de la Moneda, el Museo Botero y la Biblioteca Luis Ángel Arango.

Una de las propuestas más emblemáticas del Plan Zonal del Centro es la conocida como Manzana 5, un gran proyecto de iniciativa pública $\left(5.800 \mathrm{~m}^{2}\right)$ para consolidar la vocación turística y cultural del centro de la
Figura $\mathrm{N}^{\circ} 3$

Biblioteca El Tintal

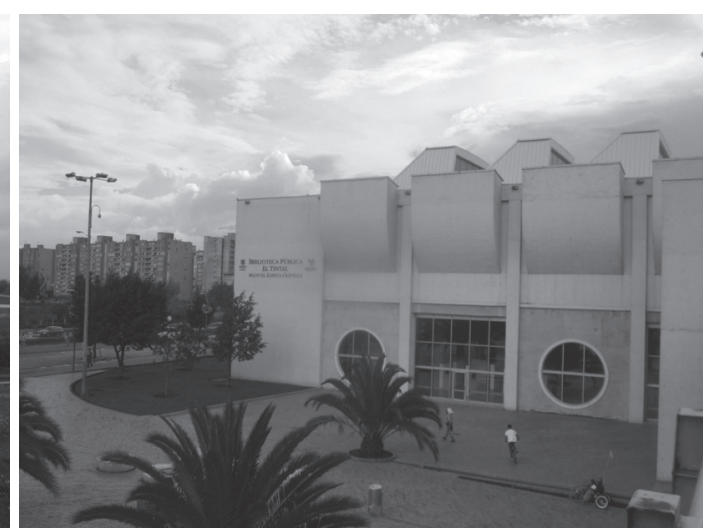

Fuente: Manuel Velandia, 2012. 
Figura $\mathrm{N}^{\circ} 4$

Centro Cultural Gabriel García Márquez

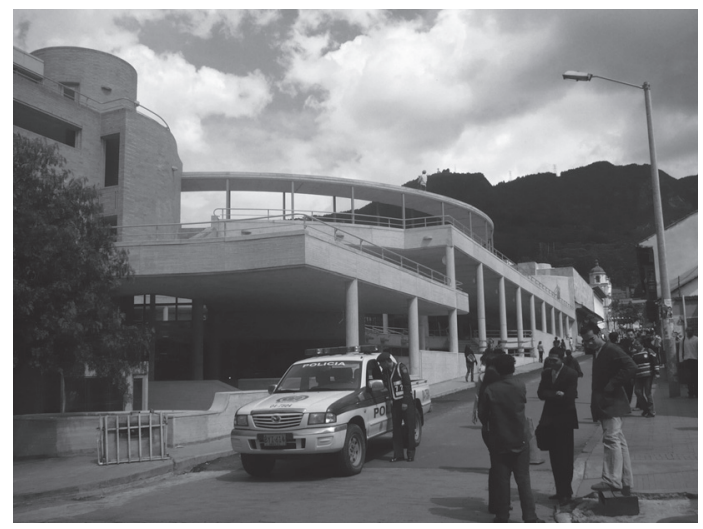

Fuente: Isabel Duque Franco, 2012.
Figura $\mathrm{N}^{\circ} 5$

Predios de Manzana 5

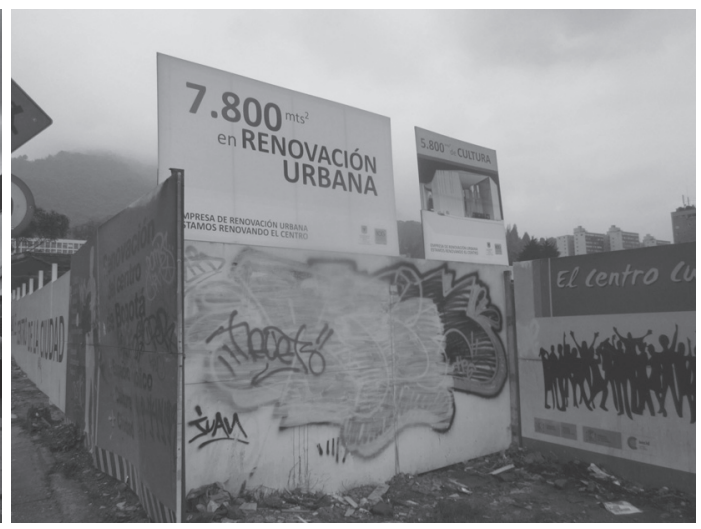

Fuente: Manuel Velandia, 2012.

en 2014, con el anuncio de la construcción de la Nueva cinemateca y centro de cultura digital de Bogotá, fue reactivado el proyecto, ratificando su dimensión cultural.

\section{Estrategias de promoción internacional}

Bogotá cuenta con una estrategia de mercadeo de ciudad, en la que venía trabajando desde 2008, en el marco de una alianza público-privada. La estrategia que tiene como objetivo "posicionar a Bogotá internacionalmente y convertirla en una ciudad líder en desarrollo humano, atractiva para el talento creativo, la inversión y el turismo" ${ }^{\prime 6}$, ha establecido entre sus metas, promover la ciudad como un centro de negocios innovadores, de industrias creativas y como capital cultural (Comité asesor, 2013). Esta apuesta articula las iniciativas de internacionalización de la ciudad, que ya se venían desarrollando, en las cuales también aparecía la cultura, aunque ahora se suman las industrias creativas.

La instrumentalización de la cultura en la promoción internacional de Bogotá, se ha hecho a través de dos estrategias: mediante la vinculación en redes de ciudades asociadas a la cultura y con la organización de eventos

\footnotetext{
6 Según el ranking de mejores ciudades para hacer negocios en América Latina de la Revista América Economía, Bogotá pasó de la posición 14 en 2004, a la número 6 en 2014.
}

\footnotetext{
4 Se trata de la tradicional Plaza de Toros la Santamaría que ha sido reconvertida en un espacio cultural, después de que la actual administración en 2012 prohibiera las ruedas de toros en la ciudad.

5 La inauguración del Centro Cultural de España en el barrio San Telmo de Buenos Aires también se vio afectada por la crisis económica española.
} 
internacionales relacionados con el sector. Como parte de la primera estrategia, Bogotá es cofundadora de la Red interlocal por la cultura que tiene como fin la formulación e implementación continental de la Agenda 21, un marco de referencia para la planificación estratégica de la cultura aprobada en el IV Foro de Autoridades Locales realizado en Barcelona en 2004 (Alcaldía Mayor de Bogotá, 2011). En 2012, la Unesco reconoció a Bogotá como una de las 69 ciudades en el mundo que integran la Red de Ciudades Creativas ${ }^{7}$, en la categoría de ciudad de la música por la fortaleza de los festivales gratuitos y al aire libre como Rock al parque que se realiza desde 1995. Por último, en 2013, Bogotá entra en el World Culture Cities Forum (WCCF), una plataforma liderada por la Alcaldía de Londres y la Autoridad del Gran Londres, integrada por 22 capitales culturales ${ }^{8}$, que tiene entre sus objetivos identificar las fortalezas y debilidades de las ciudades en materia de cultura, diseñar estrategias para promover las industrias creativas como impulso al crecimiento económico y, por supuesto, promocionar las ciudades y sus estrategias culturales en el escenario global para atraer inversiones, talento y turismo (BOP Consulting, 2013).

Como parte de la segunda estrategia, Bogotá fue la primera ciudad latinoamericana seleccionada por la Unesco como Capital Mundial del Libro en 2007, un reconocimiento por la red de bibliotecas públicas y los programas relacionados con el fomento de la lectura como Libro al Viento ${ }^{9}$ o la iniciativa PPP paraderos para-libros para-parques ${ }^{10}$. Ese

7 Esta red fue creada en 2004 para promover la cooperación internacional entre ciudades y alentarlas a impulsar asociaciones de desarrollo conjunto en función de las prioridades de la UNESCO en materia de cultura y desarrollo. Actualmente las otras ciudades latinoamericanas que integran esta Red son Popayán, Buenos Aires, Curitiba y Florianópolis.

8 También Buenos Aires y Río de Janeiro hacen parte de esta red.

9 Distribución gratuita de libros con el propósito de difundir la cultura literaria en la ciudad y vincular a los diferentes sectores de la población bogotana en experiencias de lectura compartida, con el fin de formar lectores individuales y como una alternativa de cultura ciudadana.

10 Se trata de pequeñas bibliotecas instaladas en parques de la ciudad con el fin de promover la lectura en espacios no convencionales. mismo año, la Unión de Ciudades Capitales de Iberoamérica ( $\mathrm{UCCl}$ ) escogió a Bogotá como Capital Iberoamericana de la Cultura. Por esta doble designación, durante 2007, la ciudad organizó una serie de eventos en los que participaron escritores, investigadores, editoriales y equipos de gobierno de otras ciudades. Sin embargo, no hay estudios que permitan determinar el impacto que hayan podido tener estas designaciones y eventos en la proyección internacional de Bogotá como capital cultural y en su contribución a la economía de la ciudad.

De otro parte, desde 1987 se realiza la Feria Internacional del Libro de Bogotá, considerada una de las tres de mayor impacto en Latinoamérica. En 2014 asistieron a la Feria más de 430 mil visitantes y se cerraron negocios editoriales y acuerdos comerciales por cerca de 9,4 millones de dólares (Instituto Distrital de Turismo, 2014a). Cada dos años se realiza el Festival Iberoamericano de Teatro que en su edición de 2014 tuvo 25 países invitados y 3.182.797 espectadores de los cuales el $2 \%$ eran turistas internacionales (Instituto Distrital de Turismo, 2014b).

Igualmente, la Cámara de Comercio de Bogotá ha organizado una agenda propia de eventos y ferias internacionales para promover las industrias culturales y creativas, con el fin de potenciar su aporte al desarrollo económico de la ciudad. En esta línea se encuentran la Feria Internacional de Arte de Bogotá -ArtBo-; y más recientemente, el Bogotá Audiovisual Market y el Bogotá Music Market que funcionan como ruedas de negocios del sector.

\section{Medellín: del urbanismo social a Medellinnovation}

En los últimos años Medellín ha tratado de desprenderse del estigma internacional de ciudad violenta y construir una nueva imagen como ciudad innovadora y creativa. En 1991, fue calificada como la ciudad más violenta e insegura del mundo al alcanzar los 381 homicidios por cada cien mil habitantes, esta tasa ha ido descendiendo y en 2013 se encontraba en un 52,3 (Personería de MedeIlín, 2013). No obstante, el problema de la violencia urbana era, y aún continúa siendo, 
mucho más complejo, en la medida en que está articulado al narcotráfico, al conflicto armado y además cada vez cobran más fuerza otras expresiones de inseguridad como el desplazamiento intraurbano.

Adicionalmente, a finales de los noventa, el $70 \%$ de la población vivía en condiciones de pobreza y en algunas comunas como Popular o San Javier superaba incluso el $85 \%$ (Corporación Región, 2007). Medellín padecía una profunda crisis de gobernabilidad que se expresaba en: comunas y barrios marginales, empobrecidos, desprovistos de urbanismo, de espacios públicos, controlados por los actores armados ilegales; una institucionalidad deslegitimada por las prácticas corruptas y clientelistas y una ciudadanía debilitada (Alcaldía de Medellín, 2004).

\section{Enfoques y perspectivas en torno a la cultura}

El complicado escenario descrito, sirvió como contexto de oportunidad política para incluir la cultura en la agenda del planeamiento urbano de Medellín durante las dos últimas décadas. El estudio de las políticas urbanas evidencia que la relación entre cultura y planeamiento ha sido abordada desde tres enfoques complementarios: cultura ciudadana en términos de formación y convivencia, la cultura como mecanismo de equidad e inclusión social y la cultura como estrategia de competitividad urbana.

Como una práctica de interreferencialidad entre políticas urbanas (Peck \& Theodore, 2010), los logros y aprendizajes de la cultura ciudadana en la experiencia de Bogotá, hicieron que Medellín adoptara también ese enfoque. Inicialmente, se trataba de una particular adaptación, extremadamente amplia y confusa, que abarcaba las tradiciones, valores, aptitudes, hábitos, los principios constitucionales, el buen ejemplo, el aprendizaje del perdón y la generación de un pacto entre la ciudadanía y el Estado para la convivencia y la construcción social de la ciudad (Alcaldía de Medellín, 2001). Posteriormente, se incluyó en los instrumentos de planeación en los mismos términos en que había sido formulada en Bogotá, haciendo énfasis en la práctica social y la reflexión pública como mecanismos para construir la coherencia entre el comportamiento individual (la ética y la moral), las normas (la ley como pacto social) y el comportamiento colectivo (la cultura) (Alcaldía de Medellín, 2004). La cultura se entendió entonces como un ejercicio permanente de pedagogía ciudadana, autorregulación, respeto y solidaridad. Como estos aprendizajes se dan a través del encuentro y la interacción con otros, fue necesaria la construcción de nuevos espacios públicos que facilitaran el uso intensivo de la ciudad.

Al mismo tiempo que se hablaba de cultura ciudadana, surgía el urbanismo social como un enfoque propio de intervención territorial que combinaba la transformación física, la intervención social, la gestión institucional y la participación ciudadana (Alcaldía de Medellín, 2008). Dicho enfoque consistía

\begin{abstract}
"básicamente, en el direccionamiento de grandes inversiones en proyectos urbanísticos puntuales hacia los sectores populares de la ciudad. Argumentando que la ciudad tiene una deuda histórica con estos sectores olvidados, se ha apostado a cancelarla mediante la construcción de obras infraestructurales y arquitectónicas de alta calidad y fuerte impacto tanto estético como social" (Brand, 2010: 99).
\end{abstract}

El urbanismo social instaló la cultura y en particular, el acceso a bienes y servicios culturales para los sectores sociales marginados, como parte de la mencionada deuda histórica y como una forma de inclusión social. Este enfoque se fue enriqueciendo en la medida en que se asumió la cultura como un derecho que garantizaba la buena calidad de vida y el disfrute de la ciudad. A través del urbanismo social se construían los equipamientos para resolver el déficit de espacios culturales en la ciudad, y desde la perspectiva de los derechos se buscaba hacer de Medellín "un gran escenario", impulsando la dinamización y apropiación de dichos espacios.

Por otra parte, el enfoque de la cultura como medio de dinamización económica y proyección internacional, ha estado presente en el planeamiento de Medellín desde mediados de la década de los noventa con la formulación del "Plan Estratégico de Medellín y el Área Metropolitana 2015". En este Plan, parte 
de la misión del municipio consistía en: "proyectar la ciudad de Medellín en las corrientes mundiales de la economía y la cultura" y en su visión se propuso, entre otras cosas, hacer de Medellín "una ciudad educadora" y ser el "epicentro de las políticas sociales y culturales en América Latina" (CIDEU, 2004: 253).

Estas apuestas han sido ratificadas en el Plan de desarrollo cultural 2011-2020, formulado con el apoyo del Ayuntamiento de Barcelona, la Agenda 21 de la Cultura y la Agencia Española de Cooperación. El plan tiene como objetivo "Promover y consolidar a Medellín como un espacio cultural abierto al mundo, equitativo, incluyente, deliberativo y diverso que promueve la implementación de políticas culturales participativas al servicio de la consolidación de la ciudadanía cultural" (Alcaldía de Medellín \& Universidad de Antioquia, 2011: 62). En términos generales, el plan busca articular dos perspectivas con las que ha sido abordada la cultura en la ciudad: como mecanismo de inclusión y como estrategia de la ciudad competitiva.

Así, de cara a la ciudadanía el plan busca la democratización de la producción y el consumo cultural, brindando las condiciones para que los pobladores de la ciudad puedan "crear, difundir y hacer circular sus producciones culturales" y al mismo tiempo acceder a la oferta de bienes y servicios culturales ${ }^{11}$. Mientras que de cara al mercado y a la proyección internacional, el plan apuesta por el posicionamiento de Medellín como productora cultural y por su inserción en la economía de la cultura mundial, a través de estrategias diversas como la vinculación de los sectores culturales con otros procesos de internacionalización de la ciudad, ligados al turismo de negocios, ferias y convenciones para que de esta manera la cultura pueda beneficiarse de

\footnotetext{
11 Para democratizar el acceso a la cultura y crear espacios públicos, desde la Alcaldía se ha establecido como política subsidiar el ingreso gratuito para varios museos y centros culturales de la ciudad, en otros espacios para niños menores de 12 años, adultos mayores y en general personas de los estratos socio económicos más bajos. Esta iniciativa, que había sido implementada desde 2005 por el Museo de Antioquia, supuso un incremento del $1000 \%$ en la cantidad de visitantes del museo (Alcaldía de Medellín \& BID, 2009).
}

la inversión de capital (Alcaldía de Medellín \& Universidad de Antioquia, 2011).

\section{Repertorios del planeamiento cultural urbano}

Siguiendo las tendencias globales y al igual que en Bogotá, en Medellín, las diferentes formas de abordar la cultura se han traducido en intervenciones urbanísticas como la construcción de nuevos equipamientos, aunque fundamentalmente en zonas periféricas de la ciudad, y el diseño de corredores culturales, que han contribuido a la configuración de una nueva morfología de la ciudad (Figura $\mathrm{N}^{\circ}$ 6).

Medellín cuenta hoy día con una serie de espacios públicos y equipamientos culturales considerados de calidad en términos de diseño y de oferta de servicios. Los más significativos en la nueva imagen de la ciudad son el Centro de Desarrollo Cultural Moravia y los ocho parques-biblioteca, la mayoría construidos en comunas y corregimientos que tradicionalmente habían estado marginadas del planeamiento urbano. De esta manera el gobierno urbano pretende encarar las tensiones sociales de la periferia con proyectos estratégicos encaminados a convertir los espacios públicos en escenarios para la cultura, el entretenimiento y la educación. En términos del ex alcalde Sergio Fajardo, con la construcción de estos equipamientos se trataba de "cambiarle la piel a la ciudad" y generar nuevos espacios públicos para el encuentro, la ciudadanía y la solidaridad (Fajardo, 2008).

La mayoría de estos nuevos equipamientos culturales, son resultado de procesos de renovación urbana en zonas periféricas de la ciudad, sustentados en la consigna "lo mejor para los más humildes" como una "forma de hacer justicia social, Ilevando por primera vez las inversiones grandes en términos económicos y de obras públicas a las comunidades que nunca les había tocado" (Alcaldía de Medellín \& BID, 2009: 125). El Centro de Desarrollo Cultural Moravia, obra de Rogelio Salmona, es emblemático, porque se trata de un equipamiento de calidad en un barrio de autoconstrucción, asentado sobre un antiguo basurero, en una zona central y estratégica de Medellín por su conexión con el centro histó- 
Figura $\mathrm{N}^{\circ} 6$

Localización de equipamientos culturales en Medellín

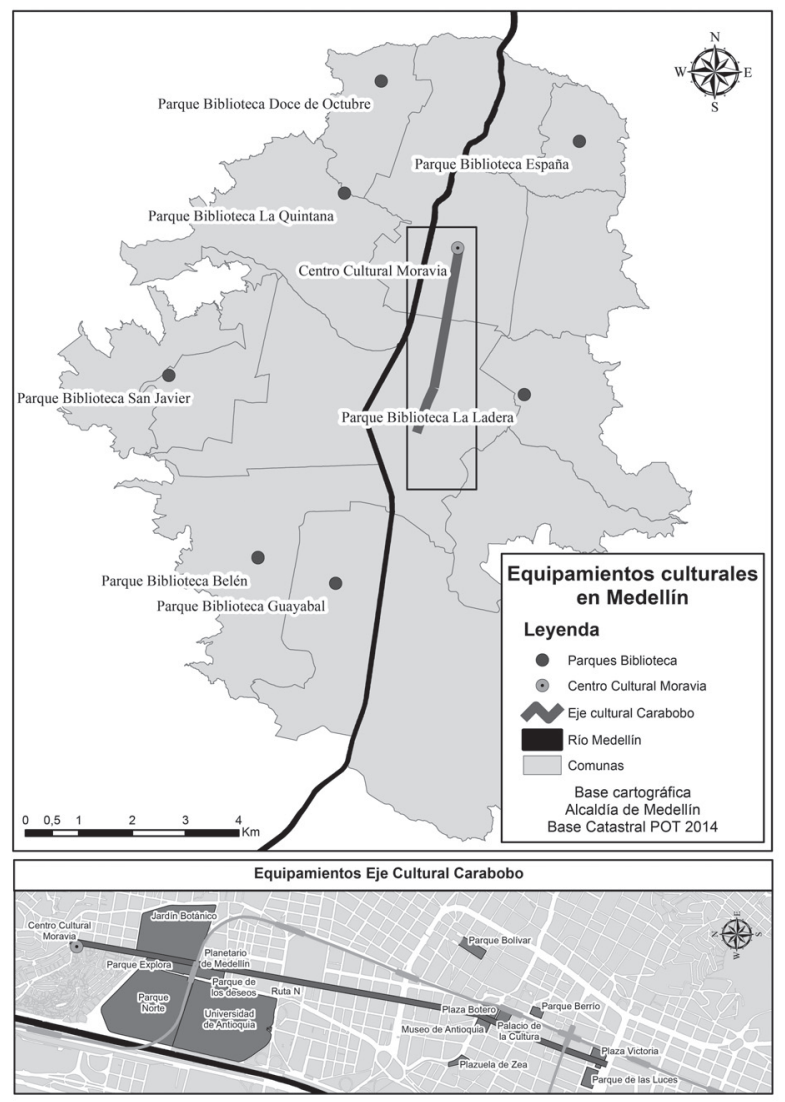

Fuente: Elaborado por Wendy González.

rico a través del Eje Carabobo. Este centro ha permitido configurar una nueva centralidad en el barrio Moravia y se ha convertido en un referente de gestión cultural, pero sobre todo, de integración social para los pobladores de la zona (Figura $N^{\circ} 7$ ).

Estos nuevos equipamientos cívico-culturales son espacios multifuncionales (plazas, terrazas, espacios para la música, la lectura, el teatro, el cine y las artes plásticas), que han tenido efectos en diferentes escalas. En la escala barrial los parques-biblioteca, junto con otras intervenciones en materia de movilidad y espacio público, han configurado áreas de centralidad periférica asociadas a la cultura. En la escala urbana estos espacios se han convertido en hitos arquitectónicos, diseñados funcional y estéticamente acorde con políticas culturales y como un referente simbólico y urbanístico para la ciudad. También estos escenarios han contribuido a la resignificación de diferentes espacios: la Biblioteca España (Figura $N^{\circ} 8$ ), construida en una de las comunas más afectadas por la violencia en la ciudad, es hoy el referente de la nueva imagen de la ciudad y lugar de peregrinación de todo tipo de visitantes y turistas $^{12}$. El Par-

12 Este Parque Biblioteca ha recibido, entre otros, el premio al diseño en la VI Bienal Iberoamericana de Arquitectura en Lisboa 2007 y el premio de la XVI Bienal Panamericana de Arquitectura de Quito en 2008. Esta obra y otra del mismo arquitecto (Giancarlo Mazzanti) realizada en Bogotá, han sido incluidas en la colección permanente del Museo de Arte Moderno de Nueva York (MoMA). Sin embargo, en 2013 apenas seis años después de haber sido 
Figura $\mathrm{N}^{\circ} 7$

Interior del Centro de Desarrollo Cultural Moravia

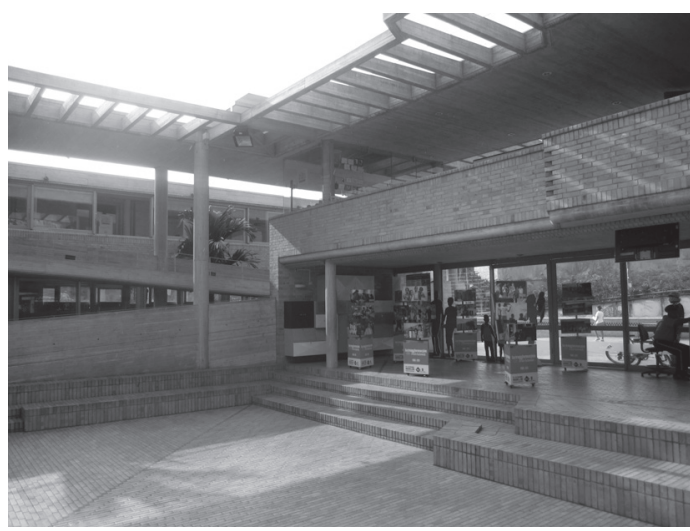

Fuente: Isabel Duque Franco, 2012.

que biblioteca Belén se construyó en lo que había sido una estación de policía y oficinas de inteligencia del ejército, asimismo, el Parque biblioteca La Ladera se levantó en los predios de la cárcel del mismo nombre, que había funcionado hasta los años sesenta.

Por otra parte, en el marco de procesos de recuperación del centro, el eje Carabobo, gran conector del centro tradicional con los desarrollos de la Alpujarra y con la zona norte de Medellín, se consolida como un corredor cultural. Se trata de una vía histórica de valor patrimonial y cultural, que desde finales del siglo XIX ha funcionado como elemento articulador de la estructura de la ciudad en términos urbanos y funcionales. Ha servido de emplazamiento de edificaciones institucionales, comerciales y culturales de alcance metropolitano, que lo han convertido en un referente de la historia de la ciudad, y más recientemente, en el escenario en donde se perfila la ciudad cultural e innovadora con proyección internacional (Morales Ramírez, 2009).

Las intervenciones sobre Carabobo y su configuración como paseo urbano y corredor cultural, hacen parte de lo que se conoce como el "Nuevo norte de la ciudad", un conjunto de proyectos locales que según el

inaugurada, el edificio entró en rehabilitación por desprendimientos en la fachada.
Figura $\mathrm{N}^{\circ} 8$

Parque Biblioteca España

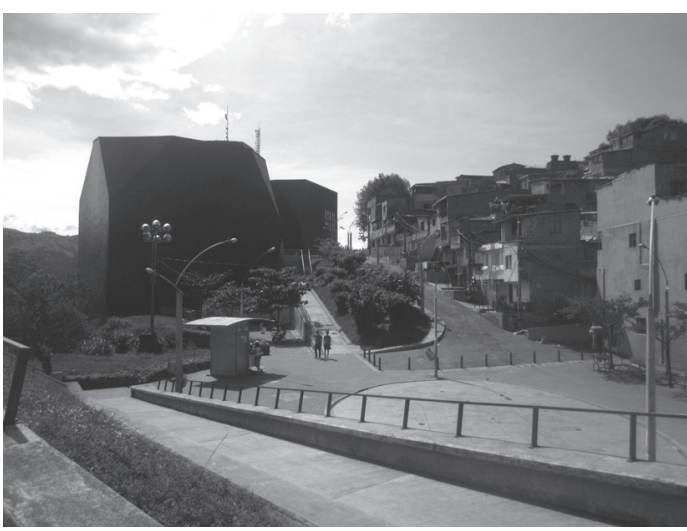

Fuente: Isabel Duque Franco, 2013.

Plan de Ordenamiento Territorial (POT) "contribuyen a la consolidación de la plataforma competitiva metropolitana" (Morales Ramírez, 2009). El paseo urbano Carabobo tiene una extensión de 4.750 metros que van desde el Puente de Guayaquil sobre el río Medellín al sur, hasta el Centro de Desarrollo Cultural Moravia al norte. A lo largo del paseo se encuentran una serie de edificaciones como testimonio de los diversos momentos y formas arquitectónicas que han estado vigentes en la ciudad (Fajardo, 2005). Al mismo tiempo, se pueden identificar unos hitos arquitectónicos y funcionales como el conjunto monumental del Museo de Antioquia, el Palacio de la Cultura y la plaza de las esculturas de Botero; o el hito univer-ciudad, conformado por la Universidad de Antioquia, la Casa de la Música, el Parque interactivo de los deseos, el Parque Explora ${ }^{13}$, el Planetario y el jardín botánico (Figura $\mathrm{N}^{\circ}$ 9).

Sin embargo, el hito más reciente lo constituye el complejo Ruta N, expresión arquitectónica de las aspiraciones de Medellín por consolidarse como una ciudad creativa, del conocimiento y de la innovación (Figura $\mathrm{N}^{\circ}$ 10). Ruta $N$ ha sido el punto de partida para la construcción del distrito de innovación Medellinnovation, un ambicioso proyecto de renovación urbanística $(168,61$ ha), ins-

\footnotetext{
${ }^{13}$ Estos tres proyectos se construyeron entre 2004 y 2007.
} 
Figura $\mathrm{N}^{\circ} 9$

Eje Carabobo

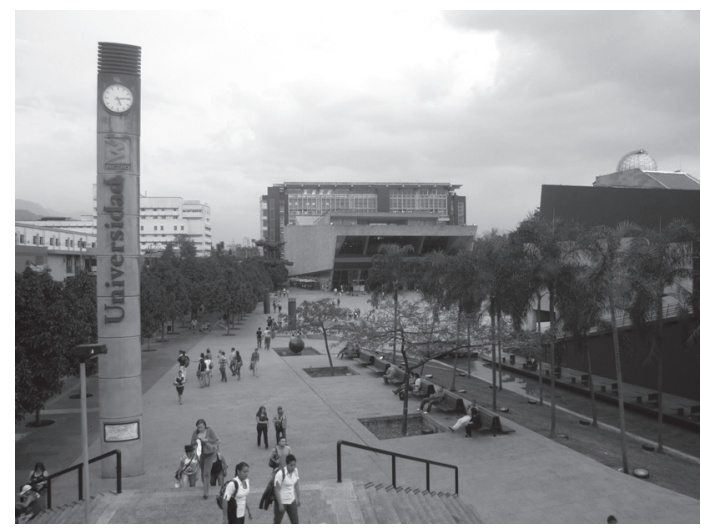

Fuente: Isabel Duque Franco, 2012.
Figura $\mathrm{N}^{\circ} 10$

Complejo Ruta $\mathrm{N}$

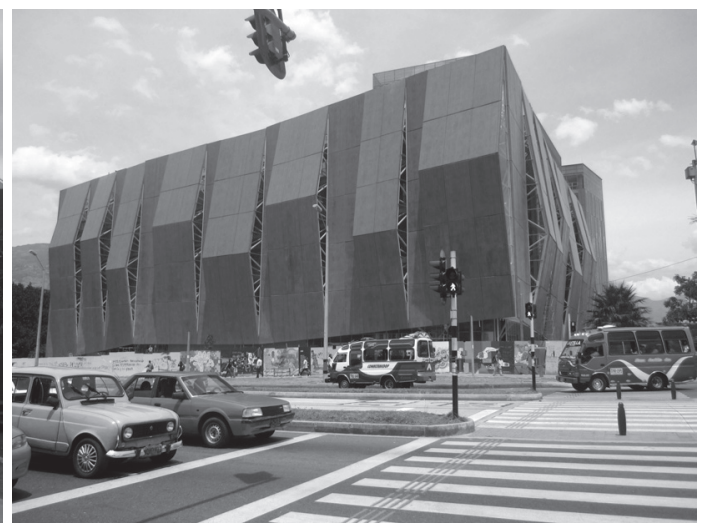

Fuente: Isabel Duque Franco, 2012. pirado en el Barcelona $22 @ 14$. A través de una alianza público-privada busca convertir el norte de la ciudad en un cluster donde se integren la ciencia, la tecnología y la innovación y que aglutine emprendedores, compañías e instituciones de la economía del conocimiento. Dentro de este proyecto, cuya consolidación está prevista para el 2023, el corredor cultural Carabobo aparece como la principal calle del Distrito (Ruta N, s.f.). Dada la magnitud y características del proyecto, es previsible que en el mediano y largo plazo, acabe generando procesos de gentrificación, tal y como ha ocurrido en Barcelona (Narrero, 2003).

En Medellín, a diferencia de Bogotá, no existe una estrategia específica para su promoción e internacionalización como ciudad cultural $^{15}$. En las campañas de difusión sobre la transformación de Medellín, promovidas por la propia administración de la ciudad o por organismos internacionales como UNHabitat o el BID (Alcaldía de Medellín \& BID, 2009; 2014), los referentes culturales apare-

${ }^{14}$ El Ayuntamiento de Barcelona asesoró a Medellín en la formulación del proyecto y en abril de 2014 los alcaldes de ambas ciudades firmaron un convenio de hermandad para la innovación y la transferencia de conocimiento.

15 Según el ranking de mejores ciudades para hacer negocios en América Latina de la Revista América Economía, Medellín pasó de la posición 36 en 2004, a la número 17 en 2014. cen como un componente más junto a la movilidad (sistema Metrocable), la intervención en zonas pobres y marginadas a través de los Proyectos Urbanos Integrales o la disminución de la criminalidad.

Asimismo, varios de los premios y reconocimientos que ha recibido la ciudad durante los últimos años, como el City to City Barcelona FAD Award (2009) o el de la Ciudad más innovadora del mundo según el concurso City of the year que organiza The Wall Street Journal, el Urban Land Institute y Citigroup (2013), coinciden en destacar las soluciones culturales dirigidas a los sectores más pobres (Parques Biblioteca y Centro de Desarrollo Cultural Moravia).

De otra parte, desde el Plan de Desarrollo Cultural, se insiste en la necesidad de insertar a Medellín en la economía de la cultura a nivel mundial por una doble vía: mediante el posicionamiento de la ciudad como productora cultural y receptora de eventos de gran resonancia internacional. Y a través de alianzas e intercambios con otras ciudades latinoamericanas como Monterrey, Mendoza, Córdoba o São Paulo, que están haciendo equipamientos culturales e importantes procesos de desarrollo cultural (Alcaldía de Medellín \& Universidad de Antioquia, 2011).

En términos de eventos, Medellín ha ampliado y diversificado su oferta cultural con actividades dirigidas a la población local y 
otras orientadas a la atracción de turistas y visitantes. Además, a las tradicionales citas como la Feria de las flores o el Desfile de mitos y leyendas, se han sumado unas nuevas como el Festival internacional Altavoz (2004), similar a Rock al parque en Bogotá, la Fiesta de las artes escénicas (2004), la Fiesta del libro y la cultura (2007), y más recientemente el Hay Festival Medellín (2015). Como eventos internacionales relacionados con el sector, vale la pena destacar el III Congreso Iberoamericano de Cultura (2010) dedicado a la música, promovido por la Organización de Estados Iberoamericanos, entre otras entidades y las $\mathrm{V}$ Jornadas Internacionales de Ciudades Creativas (2012), coordinadas por la Fundación Kreanta, una de las entidades de cooperación más comprometidas con la difusión internacional de la transformación de Medellín y promotora de las ciudades creativas en América Latina ${ }^{16}$.

En términos generales, la ausencia de una estrategia definida de promoción de Medellín como ciudad cultural, contrasta con la cantidad y diversidad de iniciativas orientadas a la atracción de inversiones y empresas con énfasis en I+D y a su posicionamiento y consolidación como "Capital de la Innovación de Latinoamérica en el 2021".

\section{Consideraciones finales}

La transformación experimentada por Bogotá y Medellín durante los últimos años resulta incuestionable, aunque en ninguno de los casos podría atribuirse completamente al factor cultural, es evidente que las iniciativas de este tipo, al ser articuladas con otras estrategias urbanas, han contribuido de manera significativa a dicha transformación. En este artículo se ha visto cómo, de la misma mane-

\footnotetext{
16 La Fundación Kreanta a través de sus proyectos de cooperación al desarrollo busca fomentar las relaciones entre ciudad, educación y cultura. Las últimas tres ediciones de las jornadas internacionales de ciudades creativas, organizadas por la Fundación, se han Ilevado a cabo en Medellín (2012), Buenos Aires (2013) y Monterrey (2014). Esto sumado a la diversidad de experiencias de ciudades latinoamericanas presentadas en estos eventos, ilustran la centralidad que ha ido tomando la cultura y la creatividad en la planeación de estas ciudades. (http://2014.ciudadescreativas.org/)
}

ra que la unión entre cultura y planeamiento urbano surge en un momento de crisis y de reestructuración económica, en las ciudades colombianas, la cultura aparece como respuesta a unos contextos críticos en materia de seguridad y convivencia, de exclusión y gobernabilidad. Por eso, la cultura ciudadana en tanto nueva gramática social, adoptada en ambos casos, resulta fundamental y permite delinear el camino hacia la redefinición de la imagen de las dos ciudades; una condición necesaria para sus aspiraciones de entrar en el escaparate mundial de las ciudades en movimiento, en donde ocurren cosas.

Además de la cultura ciudadana, los enfoques y narrativas que han orientado el planeamiento cultural en Bogotá y Medellín, están asociados a la cultura como derecho, como mecanismo de inclusión social y al mismo tiempo como estrategia de competitividad urbana y proyección internacional. Así, en ambos casos la inclusión de la cultura ha servido para configurar un modelo de ciudad y de gobernanza urbana, que si bien hace explícito el cambio de rumbo del desarrollo urbano, evidencia las tensiones que supone tratar de situar a los ciudadanos en el centro de las prioridades y al mismo tiempo acondicionar la ciudad para su inserción en las redes de la economía global.

Esta diversidad y riqueza de los enfoques que han inspirado el planeamiento cultural urbano en Bogotá y Medellín, ilustran las especificidades del proceso en estas ciudades (Cuadro $\mathrm{N}^{\circ} 1$ ). No pareciera ocurrir lo mismo con los repertorios de intervención urbana, dado que ambas ciudades recurren a fórmulas muy semejantes; los procesos de renovación urbana, la articulación de corredores culturales y la construcción de bibliotecas como equipamiento cultural básico o "centros de atención cultural primaria" (Manito, 2006: 12). No obstante, alrededor de estos proyectos se han generado ciertas dinámicas que vale la pena reseñar: primero, la reactivación de los barrios y la formación de centralidades periféricas; segundo, la existencia de procesos de renovación urbana diferenciados, en Bogotá más orientado a articular la infraestructura cultural existente a través de un corredor, mientras que en Medellín la articulación de lo existente se complementa con nueva infraestructura, consolidando un 
Cuadro $\mathrm{N}^{\circ} 1$

Planeamiento urbano cultural: síntesis comparativa

\begin{tabular}{|c|c|c|}
\hline Categorías & Bogotá & Medellín \\
\hline $\begin{array}{l}\text { Enfoques y perspectivas } \\
\text { de la cultura }\end{array}$ & $\begin{array}{l}\text { Cultura ciudadana } \\
\text { Escala humana } \\
\text { Derechos } \\
\text { Generadora de riqueza } \\
\text { Estrategia de competitividad }\end{array}$ & $\begin{array}{l}\text { Cultura ciudadana } \\
\text { Inclusión } \\
\text { Estrategia de competitividad }\end{array}$ \\
\hline $\begin{array}{l}\text { Repertorios/ } \\
\text { intervenciones } \\
\text { urbanísticas }\end{array}$ & $\begin{array}{l}\text { Equipamientos de escala zonal y } \\
\text { urbana } \\
\text { Renovación urbana } \\
\text { Corredor cultural/articulación de } \\
\text { lo existente }\end{array}$ & $\begin{array}{l}\text { Equipamientos en zonas perifé- } \\
\text { ricas } \\
\text { Renovación urbana } \\
\text { Corredor cultural / nueva cons- } \\
\text { trucción } \\
\text { Distrito (industrial) de innovación }\end{array}$ \\
\hline Estrategias de promoción & $\begin{array}{l}\text { Campaña de mercadeo de ciudad } \\
\text { Redes de ciudades } \\
\text { Agenda de eventos } \\
\text { Ruedas de negocios }\end{array}$ & $\begin{array}{l}\text { Premios y reconocimientos } \\
\text { Agenda de eventos }\end{array}$ \\
\hline
\end{tabular}

Fuente: Elaboración propia.

corredor que se convierte en pieza central de un proyecto de mayor impacto que ensambla cultura, creación e innovación.

En síntesis, la incorporación de la cultura en el planeamiento urbano de las dos ciudades se ha hecho visible en el espacio urbano y ha servido para la circulación a nivel internacional de imágenes e idearios de ciudad en donde se muestra, la cultura en Bogotá y la innovación en Medellín, como motores de cambio y transformación. La difusión internacional de esta imagen de ciudades transformadas desde la cultura, las ha convertido, antes a Bogotá y ahora a Medellín, en referentes de planeación y gobernanza urbana, sedes de grandes eventos, ganadoras de todo tipo de premios y reconocimientos (por supuesto gracias a campañas de marketing y candidaturas cuidadosamente diseñadas), al tiempo que atraen turistas, inversores y escalan posiciones en la jerarquía urbana global, como si de un "círculo virtuoso" se tratara. De esta manera, las dos ciudades han tomado la tendencia global de la instrumentalización de la cultura en el planeamiento urbano, la han adaptado con retóricas propias y diversas aunque con prácticas más bien estandarizadas que ahora se reproducen y amplían en otros contextos.

\section{Referencias Bibliográficas}

ALCALdía MAYOR DE BOGOTÁ D.C. Plan Maestro de Equipamientos Culturales. Bogotá: Alcaldía Mayor de Bogotá D.C., 2006.

AlCALdía MAYOR DE BOGOTÁ D.C. Políticas culturales distritales 2004-2016. Bogotá: Alcaldía Mayor de Bogotá D.C., 2005.

ALCALdía MAYOR DE BOGOTÁ D.C. Plan Decenal de Cultura Bogotá D.C. 20122021. Bogotá: Alcaldía Mayor de Bogotá D.C., 2011.

AlCALDía DE MEDELlíN. Plan de Desarrollo 2001 - 2003 Medellín Competitiva. Medellín: Alcaldía de Medellín, 2001.

ALCALDÍA DE MEDELLíN. Plan de Desarrollo 2004-2007 "Medellín compromiso de toda la ciudadanía". Medellín: Alcaldía de Medellín, 2004.

AlCALDíA DE MEDELlíN. Plan de Desarrollo 2008-2011 "Medellín es solidaria y competitiva". Medellín: Alcaldía de Medellín, 2008. 
ALCALDÍA DE MEDELLÍN \& UNIVERSIDAD DE ANTIOQUIA. Plan de Desarrollo Cultural de Medellín 2011-2020. Medellín: Alcaldía de Medellín, 2011.

ALCALDÍA DE MEDELLÍN \& BID. Medellín: transformación de una ciudad. Medellín: Alcaldía de Medellín \& Banco Interamericano de Desarrollo, 2009.

ARANTES, O. Uma estratégia fatal. A cultura das novas gestões urbanas. In: ARANTES, O, VAINER, C. \& MARICATO, E. A cidade do pensamento unico. Desmanchando consensos. Rio de Janeiro: Vozes, 2000, p.11-74.

BATTAGLIA, A. \& TREMBLAY, D. El Raval and Mile End: A Comparative Study of Two Cultural Quarters between Urban Regeneration and Creative Clusters. Joumal of Geography and Geology, 2012, Vol. 4, № 1, p. 56-74.

BAYLISS, D. Creative planning in Ireland: The role of culture-led development in irish planning. European Planning Studies, 2004, Vol. 12, No 4, p. 497-515.

BAYLISS, D. The Rise of the Creative City: Culture and Creativity in Copenhagen. European Planning Studies, 2007, Vol. 15, № 7, p. 889-903.

BENNEWORTH, P. \& DAUNCEY, H. International urban festivals as a catalyst for governance capacity building. Environment and Planning C: Government and Policy, 2010, Vol. 28, p. 1083-1100.

BEUF, A. De las luchas urbanas a las grandes inversiones. La nueva urbanidad periférica en Bogotá. Bulletin de L'Institut Francais D'Etudes Andines, 2012, Vol. 41, N 3, p.473-501.

BOP CONSULTING. World cities culture report. London: Mayor of London, 2013.

BRAND, P. El 'urbanismo social' en MedeIlín, Colombia. Revista Arquitectura COAM, 2010, No359, p. 99-104.

CAPEL, H. El modelo Barcelona: un examen crítico. Barcelona: Serbal, 2005.
CASTRO COMA, M. Del sueño olímpico al proyecto Porto Maravilha: el 'eventismo' como catalizador de la regeneración a través de grandes proyectos urbanos. Urbe. Revista Brasileira de Gestão Urbana, 2011, Vol. 3, № 2, p. 211-227.

CIDEU. 10 años de planificación estratégica en Iberoamérica. Barcelona: CIDEU, 2004.

COMITÉ ASESOR. Bogotá, estrategia de mercadeo de ciudad. Bogotá, 2013.

CONCEJO DE BOGOTÁ. Acuerdo 6 de 1998 . Plan de Desarrollo Económico, Social y de Obras Públicas para Santa Fe de Bogotá, D.C., 1998 - 2001 "Por la Bogotá que queremos". Bogotá, 1998.

CORPORACIÓN REGIÓN. Panorama social de Medellín. Diagnóstico social de Medellín y evaluación del modelo de intervención de la Secretaría de Bienestar Social - 2007. Medellín: Corporación Región y Alcaldía de Medellín, 2007.

CUENYA, B. y CORRAL, M. Empresarialismo, economía del suelo y grandes proyectos urbanos: el modelo de Puerto Madero en Buenos Aires. EURE, 2011, Vol. 37, №111, p. 25-45.

DEVESA, M.; BÁEZ, A.; FIGUEROA, V. \& HERRERO, L. Repercusiones económicas y sociales de los festivales culturales: el caso del Festival Internacional de Cine de Valdivia. EURE, 2012, Vol. 38, № 115, p. 95-115.

DUQUE FRANCO, I. Planeamiento urbano en Bogotá 1994-2007. La construcción de un modelo. Scripta Nova. Revista Electrónica de Geografía y Ciencias Sociales, 2008, Vol. XII, No 270 (57). Disponible en Internet: $<$ http://www.ub.es/geocrit/sn/sn-270/sn-27057.htm>

EVANS, G. Hard-Branding the Cultural City from Prado to Prada. International Journal of Urban and Regional Research, 2003, Vol. 27, No 2, p. 417-40.

EVANS, G. \& SHAW, P. The Contribution of Culture to Regeneration in the UK: A Report to the Department for Culture, Media 
and Sport. London: London Metropolitan University, 2004.

FAJARDO, S. El Paseo Carabobo, un proyecto de ciudad. Medellín: Centr@dentro No 1, Alcaldía de Medellín, 2005.

FAJARDO, S. Cambiarle la piel a la ciudad. En: BELTRÁN, R. y MANITO, F. Aprendiendo de Colombia: Cultura y educación para transformar la ciudad. Bogotá: Fundación Kreanta y CAB, 2008, p. 168-174.

FLORIDA, R. Cities and the creative class. London/New York: Routledge, 2005.

GARCÍA, B. Urban regeneration, arts programming and major events Glasgow 1990, Sidney 2000 and Barcelona 2004. International Journal of Cultural Policy, 2004, Vol. 10, $\mathrm{N}^{\circ} 1$, p. 103-118.

GOLD, J. \& WARD, S. Place promotion: the use of publicity and marketing to sell towns and regions. Chichester: Wiley \& Sons Publishers, 1994.

GÓMEZ, M.V. Reflective images: the case of urban regeneration in Glasgow and Bilbao. International Journal of Urban and Regional Research, 1998, Vol. 23, N³, p. 589-92.

GONZÁLEZ, S. The role of the Guggenheim Museum in the development of urban entrepreneurial practices in Bilbao. International Journal of Iberian Studies, 2004, Vol. 16, N 3, p. 177-186.

HARVEY, D. From managerialism to entrepreneurialism: the transformation in urban governance in late capitalism. Geografiska, 1989, Vol. 71B, p. 3-17.

INSTITUTO DISTRITAL DE TURISMO. Investigación de asistentes a eventos de ciudad. Feria Internacional del libro de Bogotá. Bogotá: Alcaldía Mayor de Bogotá, 2014.

INSTITUTO DISTRITAL DE TURISMO. Investigación de asistentes a eventos de ciudad. Festival Iberoamericano de Teatro. Bogotá: Alcaldía Mayor de Bogotá, 2014 b.

JANOSCHKA, M. y SEQUERA, J. Procesos de gentrificación y desplazamiento en Amé- rica Latina, una perspectiva comparativista En: MICHELINI, J.J. (editor). Desafíos metropolitanos. Un diálogo entre Europa y América Latina. Madrid: Catarata, pp. 82-104.

LANDRY, C. \& BIANCHINI, F. The creative city. London: Demos, 1995.

LANDRY, C. The Creative City: A Toolkit for Urban Innovat. London: Earthscan Publications, 2000.

LIMA, C. Una mirada crítica a la zona portuaria de Río de Janeiro. Revista Bitácora Urbano Territorial, 2010, Vol. 17, №2, p. 23-54.

MANITO, F. Cultura y estrategia de ciudad. La centralidad del sector cultural en la agenda local. Barcelona: CIDEU, 2006.

MANITO, F. (editor). Ciudades creativas. Volumen 3. Economía creativa, desarrollo urbano y políticas públicas. Barcelona: Fundación Kreanta, 2011.

MARTÍN, G. y CEBALLOS, M. Bogotá: Anatomía de una transformación. Políticas de seguridad ciudadana 1995-2003. Bogotá: USAID, Universidad de Georgetown, Alcaldía Mayor de Bogotá y Universidad Javeriana, 2004.

MÉNDEZ, R.; MICHELINI, J.J.; PRADA, J. y TÉBAR, J. Economía creativa y desarrollo urbano en España: una aproximación a sus lógicas espaciales. EURE, 2012, Vol. 32, N 113, p. 5-32.

MOCKUS, A. Conciliar ley, moral y cultura. En: VEEDURÍA DISTRITAL. Participación ciudadana en la planeación del desarrollo municipal, distrital y nacional. Bogotá: Veeduría Distrital, 2001, p. 17-35.

MORALES RAMÍREZ, N. Estrategias discursivas de control social en la planeación del espacio público del centro de Medellín: Eje Carabobo 2004-2007. Medellín: Universidad Nacional de Colombia, sede Medellín, Maestría en Estudios Urbano Regionales, Escuela de Planeación Urbano Regional, 2009.

MUÑOZ, F. Urbanalización. Paisajes comunes, lugares globales. Barcelona: Gustavo Gili, 2008. 
NARRERO, I. ¿Del Manchester catalán al Soho Barcelonés? La renovación del barrio del Poble Nou en Barcelona y la cuestión de la vivienda. Scripta Nova. Revista electrónica de geografía y ciencias sociales, 2003, Vol. VII, No 146(137). Disponible en Internet: http://www.ub.es/geocrit/sn/sn-146(137).htm

PECK, J. \& THEODORE, N. Mobilizing policy: Models, methods, and mutations. Geoforum, 2010, № 41, p. 169-174.

PECK, J. Geographies of policy: From transfer-diffusion to mobility-mutation. Progress in Human Geography, 2011, Vol. 35, № 6, p. 773-797.

PERSONERÍA DE MEDELLÍN. Informe sobre la situación de los derechos humanos en la ciudad de Medellín, Medellín: Personería de Medellín, 2013.

PLAZA, B. The Return on Investment of the Guggenheim Museum Bilbao. International Journal of Urban and Regional Research, 2006, Vol. 30, N 2, p. 452-67.

PNUD. Bogotá una apuesta por Colombia. Informe de Desarrollo Humano. Bogotá: PNUD, 2008.
RAMON, R. Del barrio al universo. En: MANITO, F. (editor). Ciudades creativas. ECOnomía creativa, desarrollo urbano y políticas públicas. Barcelona: Kreanta, 2011, p. 199221.

RUTA N. Tecnologías, mercados y la ciudad en transformación. Medellín, s.f.

SANTAGATA, W. Cultural districts, property rights and sustainable economic growth. International Journal of Urban and Regional Research, 2002, Vol. 26, № 1, p. 9-23.

SCOTT, A. Cultural economy and the creative field of the city. Geografiska Annaler: Series B, Human Geography, 2010, Vol. 92, p. 115-130.

UN-HABITAT. Cultural strategies for urban development. In: United Nations Human Settlements Programme. The state of the world cities 2004/2005. Globalization and urban culture. London: Earthscan, 2004, p. 31-48.

ZUKIN, S. The cultures of cities. Oxford and Cambridge : Blackwell Publishers, 1995. 
